\title{
DATOS PRELIMINARES DE LA CONTRIBUCIÓN ESPAÑOLA AL ESTUDIO RANDOMIZADO EUROPEO DE SCREENING DEL CÁNCER DE PRÓSTATA (ERSPC).
}

\author{
Gina Torres Zambrano, Marcos Lujan Galán, Carlos Pascual Mateo, Ana García Tello, Nuria \\ Rodríguez y Antonio Berenguer Sánchez.
}

Servicio de Urología. Hospital Universitario de Getafe. Getafe. Madrid. España.

\begin{abstract}
Resumen.- OBJETIVO: Datos de la participación de nuestro Centro en el ERSPC en términos de detección y rendimiento diagnóstico del PSA, estadiaje, manejo terapéutico y mortalidad, en el contexto de un estudio multicéntrico randomizado de screening del cáncer de próstata (CaP).

MÉTODOS: Se reclutaron pacientes entre febrero de 1996 y junio de 1999, randomizando en brazos screening y control, realizado varias rondas a los pacientes del brazo screening con intervalo de 4 años, con determinación de PSA y biopsia prostática sextante IPSAt $>4 \mathrm{ng} / \mathrm{mL}$ hasta mayo de 1998, posteriormente PSAt $>2.99 \mathrm{ng} / \mathrm{mL}$ ), sin realizar dichos tests en los varones del grupo control. Se investigaron los fallecimientos (fecha de defunción y causa de la mismal.
\end{abstract}

Gina Torres Zambrano

Servicio de Urología

Hospital Universitario de Getafe

Ctra. Toledo. Km 12.500

28905 Getafe. Madrid. (España). gmtorres.hugf@salud.madrid.org

Trabajo recibido: 7 de marzo 2007
RESULTADOS: Se incluyeron un total de 4.278 pacientes, 2.416 del brazo screening (56,4\%) y 1.862 del brazo control (43,5\%), con edad entre 45 a 70 años y media de 57,8 años (IC95 57,6-58,0). La mediana del tiempo de seguimiento fue de 8,77 años. Se encontraron 142 cánceres, 113 del brazo screening (tasa de detección acumulada 4,7\%) y 29 del brazo control $(1,6 \%)$. Las tasas de detección fueron de 1,7\%,2,2\% y $0 \%$ en la primera, segunda y tercera ronda respectivamente. Los cánceres órgano confinados (T1 y T2) fueron 102 en el brazo screening (90,3\%) y 24 en el control $(82,8 \%), p=0,254$. Se detectaron 6 pacientes con enfermedad metastásica (4,2\% de 142), 3 del brazo screening (2,7\%) y 3 del control $(10,3 \%), p=0,187$. El tratamiento más realizado fue la prostatectomía radical: 47 del brazo screening $(41,6 \%)$ y 10 del control (34,5\%). Se han producido 151 fallecimientos, 82 en el brazo screening y 69 en el control, de estos sólo 3 fallecimientos por $\mathrm{CaP}$, todos del brazo screening ( $p=0,308)$.

CONCLUSIONES: Se observa tendencia no significativa hacia estadíos clínicos más precoces en los pacientes sometidos a screening. El tratamiento de elección en los pacientes con CaP fue la cirugía radical. Aún con el seguimiento actual de esta serie (cercano a los 9 años), no se han observado en nuestro Centro diferencias en cuanto a la mortalidad por CaP entre los dos brazos del estudio.

Palabras clave: Cáncer de próstata. Screening. Detección temprana. Prostatectomía radical. 
Summary.- OBJECTIVES: Data from the participation of our department in the ERSPC study in terms of detection and PSA diagnostic yield, staging, therapeutic management and mortality in the context of a multicentric randomized screening study for prostate cancer.

METHODS: Patients were recruited between February 1996 and June 1999, randomized in screening and control arms, with several rounds for screening patients with four-year intervals, with PSA analysis and sextant prostate biopsy (t PSA > $4 \mathrm{ng} / \mathrm{ml}$ before May 1998, tPSA > $2.99 \mathrm{ng} / \mathrm{ml}$ after May 1998). These tests were not done in the control group. All deaths were studied (date and cause)

RESULTS: A total of 4278 patients were included, 2416 in the screening arm (56.4\%) and 1862 in the control arm (43.5\%), with an age between 45 and 70 years (mean age 57.8 years, $95 \mathrm{Cl} 57.6-58.0$ ). Median follow-up was 8.77 years. 142 prostate cancers were found, 113 in the screening arm laccumulated detection rate $4.7 \%$ ) and 29 in the control arm (1.6\%). Detection rates were $1.7 \%, 2.2 \%$ and $0 \%$ in the first, second and third round respectively. Organ confined cancers (TI and T2) were 102 in the screening arm (90.3\% ) and 24 in the control arm (82.8\%) , $p=0.254$. Metastatic disease was found in six patients ( $4.2 \%$ out of 142 ), 3 in the screening arm (2.7\%) and three in the control $\operatorname{arm}(10.3 \%), p=0.187$. Radical prostatectomy was the most frequently performed treatment: 47 cases in the screening arm (41.6\%), 10 in the control arm (34. $5 \%$ ). 151 patients have died, 82 in the screening arm and 69 in the control arm. Only 3 of this deaths were secondary to prostate cancer, all of them in the screening $\operatorname{arm}(p=0.308)$.

CONCLUSIONS: We observed a non significant tendency to lower clinical stages in patients undergoing screening. Radical surgery was the treatment of choice in patients with prostate cancer. Even with the current follow-up in the series (close to 9 years), no differences have been observed in terms of prostate cancer mortality between both study arms.

Keywords: Prostate cancer. Screening. Early detection. Radical prostatectomy.

\section{INTRODUCCIÓN}

El cáncer de próstata ( $\mathrm{CaP}$ ) es una enfermedad con elevada incidencia, ya que se diagnostican anualmente unos 500.000 varones en el mundo (1). Presenta también una alta tasa de mortalidad y morbilidad. En Estados Unidos es el tumor maligno más frecuente y supone la tercera causa de muerte por cáncer en varones (2). En nuestro ámbito (área sanitaria 10 de la Comunidad de Madrid) la incidencia ajustada a la pirámide de población española fue de 87 nuevos casos / 100.000 varones durante el año 2004. El CaP afecta al 10\% de los varones a lo largo de la vida y hasta un 3\% mueren por esta causa $(3,4)$. En la era pre-PSA el CaP no palpable era raramente detectado (5) pero, desde hace dos décadas y debido al descubrimiento del PSA como marcador tumoral (6), son más diagnosticados los tumores órgano-confinados. Ello también conlleva la detección de un número mayor de tumores, aún sabiendo que es posible que muchos de ellos no necesiten de un tratamiento agresivo (fenómeno de sobredetección) $(7,8)$.

Se estima que 3 de cada 4 hombres de 50 años o más, son sometidos a determinación rutinaria de PSA $(6,9)$, debido a la tendencia de muchos países del mundo desarrollado a practicar el screening del CaP. Ello se debe en parte a las recomendaciones de las distintas sociedades científicas (10), aún sin haber sido totalmente demostrada su eficacia. Además, se sabe que el nivel de PSA sérico es un buen marcador para monitorizar la evolución de esta enfermedad $(3,11)$, pero no existe evidencia clínica concluyente de que el uso masivo de esta práctica reduzca la mortalidad por esta enfermedad en la población $(1,3)$. Para resolver esta incógnita existen estudios randomizados en marcha. Los más relevantes son el European Randomized Study of Screening for Prostate Cancer (ERSPC) en el que participan varios países europeos (entre ellos nuestro Centro), cuyos resultados se esperan a partir de 2008 con posibilidad de análisis conjunto con otro estudio, y el Prostate Lung Colorectal and Ovary study (PLCO) en Estados Unidos (12). Por último, en el Reino Unido otro estudio ha sido recientemente iniciado, el Protec-T (13), aunque sus resultados no estarán disponibles antes de un plazo más largo.

En el presente trabajo ofrecemos los datos correspondientes a la participación de nuestro Centro en el ERSPC hasta el momento actual, en términos de detección y rendimiento diagnóstico del PSA, estadiaje de los cánceres detectados así como del manejo terapéutico de los mismos. La mortalidad global y cáncer-específica serán también estudiadas.

\section{MATERIALES Y MÉTODOS}

Nuestro centro, el Servicio de Urología del Hospital Universitario de Getafe, perteneciente al área 10 de la Comunidad de Madrid, es el único centro en España que forma parte del ERSPC. Junto 
al PLCO en EEUU, es uno de los principales estudios en marcha para demostrar una reducción de la mortalidad por $\mathrm{CaP}$ entre la población de varones sometidos a screening $(3,12,14)$. Este estudio esta financiado por la Comunidad Europea para centralizar las bases de datos de 8 países europeos (Bélgica, España, Francia, Finlandia, Holanda, Italia, Suecia y Suiza), del cual se esperan los primeros resultados a partir del año 2008. En este estudio se han reclutado desde 1994 hasta la fecha actual unos 250.000 varones con edad comprendida entre 45 y 75 años. Los varones participantes han sido randomizados para su inclusión en brazo screening (realización de tests) y brazo control (no realización de tests). El objetivo principal es demostrar una reducción de la mortalidad por CaP en el brazo screening.

En nuestro Centro el proceso de reclutamiento se realizó entre febrero de 1996 y junio de 1999, con varones entre 45 a 70 años, mediante carta de invitación para participar a partir de las listas del censo de población, tras randomización en grupos de screening y control mediante ordenador, excluyendo los pacientes con CaP ya conocido, o patología concomitante que podría suponer una disminución en la esperanza de vida. Se han realizado varias rondas de screening con determinación de PSA y biopsia prostática en los casos indicados, con un intervalo de 4 años entre dichas rondas. En casos de riesgo, se realizaron visitas adicionales entre dos rondas consecutivas. No se realizaron dichos tests en los varones del grupo control. Los criterios de biopsia prostática han sufrido en nuestro Centro ligeras variaciones a lo largo del periodo de participación. Desde el inicio de la misma hasta mayo de 1998, el criterio para la toma de biopsia fue un PSA total (PSAt) $>4 n g /$ $\mathrm{mL}$. Posteriormente, se decidió bajar dicho umbral a PSAt $>2,99 \mathrm{ng} / \mathrm{mL}$ hasta nuestros días. De modo adicional (entre febrero de 2002 y mayo de 2003) se incluyó, como indicación de biopsia, los casos con PSAt $<3 \mathrm{ng} / \mathrm{mL}$, con ratio PSA libre/total $<15 \%$.
Las biopsias fueron tomadas con ayuda de ecografía transrectal por sextantes con realización simultánea de tacto rectal, sin que los hallazgos de éste constituyeran en modo alguno indicación para biopsia.

Las neoplasias detectadas se sometieron a tratamiento curativo (opción recomendada en nuestro Centro) si clínicamente localizadas, mediante prostatectomía radical y radioterapia prostática externa. La observación vigilada se llevó a cabo en los pacientes que desearon esta alternativa de modo expreso tras ser informados de las alternativas disponibles.

Por último se investigaron los fallecimientos acontecidos en los participantes reclutados en ambos brazos. Se registraron la fecha de defunción y la causa de la misma. Los fallecidos en el hospital de referencia de nuestra área (Hospital Universitario de Getafe) fueron incorporados desde los sistemas de información del Centro, tras revisión de la historia clínica. Un comité local de causas de muerte verificó que la causa finalmente registrada se correspondía con la información de la historia clínica. También se registraron los fallecimientos producidos fuera de nuestro Centro mediante convenio con el Instituto Nacional de Estadística, tomando como causa de muerte la recogida como causa fundamental en el certificado de defunción.

\section{RESULTADOS}

La población invitada en nuestro Centro fue de 18.612 varones. El total de pacientes incluidos en nuestro estudio fue de 4.278 (tasa de participación del 23\%), siendo randomizados 2.416 en el brazo screening $(56,4 \%)$ y 1.862 en el brazo control $(43,5 \%)$, con edades entre 45 a 70 años y media de 57,8 años (IC95 57,6-58,0). La distribución de ambos brazos del estudio por grupo de edad se ofrece en la Tabla I.

TABLA I. DISTRIBUCIÓN DE LA POBLACIÓN RECLUTADA POR GRUPOS DE EDAD.

\begin{tabular}{|l|l|l|l|l|l|l|l|}
\hline & $45-49$ & $50-54$ & $55-59$ & $60-64$ & $65-69$ & $70-74$ & Total \\
\hline Grupo Screening & 153 & 746 & 734 & 478 & 282 & 23 & 2416 \\
\hline Grupo Control & 177 & 526 & 481 & 385 & 275 & 18 & 1862 \\
\hline Total & 330 & 1272 & 1215 & 863 & 557 & 41 & 4278 \\
\hline
\end{tabular}




\section{TABLA II A. GRUPO SCREENING, PRIMERA RONDA. DISTRIBUCIÓN SEGÚN NIVEL DE PSA, BIOPSIAS REALIZADAS Y CÁNCERES DETECTADOS.}

\begin{tabular}{|l|c|c|c|c|c|c|}
\hline PSA en $\mathrm{ng} / \mathrm{ml}$ & $\begin{array}{c}\text { Número de } \\
\text { varones en } \\
\text { screening }\end{array}$ & $\begin{array}{c}\text { Número } \\
\text { referido }\end{array}$ & $\begin{array}{c}\text { Número de } \\
\text { biopsias }\end{array}$ & $\begin{array}{c}\text { Número de } \\
\text { CaP }\end{array}$ & $\begin{array}{c}\mathrm{P} \\
\text { de CaP } \\
(\%)\end{array}$ & $\begin{array}{c}\text { Detección } \\
\text { diferida }\end{array}$ \\
\hline$<3$ & 2152 & 0 & 0 & 0 & 0,0 & 0 \\
\hline$\geq 3<4$ & 97 & 37 & 31 & 4 & 4,1 & 6 \\
\hline$\geq 4<7$ & 115 & 112 & 90 & 19 & 16,5 & 7 \\
\hline$\geq 7<10$ & 30 & 30 & 25 & 9 & 30,0 & 2 \\
\hline$\geq 10$ & 22 & 22 & 20 & 8 & 36,4 & 2 \\
\hline Total & 2416 & 201 & 166 & 40 & 1,7 & 18 \\
\hline
\end{tabular}

El tiempo de seguimiento medio desde el reclutamiento hasta la fecha de confección del presente trabajo fue de 8,90 años (IC95 8,88-8,92), mediana de 8,77 años. Se encontraron 142 cánceres desde el inicio del estudio hasta hoy (tasa de detección acumulada 3,3\%), 113 del brazo screening (tasa de detección acumulada $4,7 \%$ ) y 29 del brazo control, (tasa de detección acumulada $1,6 \%)$. Durante la primera ronda de los pacien- tes sometidos a screening se detectaron $40 \mathrm{CaP}$ (tasa de detección del 1,7\%). El rendimiento de las biopsias realizadas así como la distribución de tumores detectados según rango de PSA y ronda del estudio se ofrecen en las Tablas IIA, IIB, y IIC. En la segunda ronda se encontraron 39 nuevos casos de cáncer de próstata (tasa de detección del 2,2\%). El la tercera ronda no se detectaron nuevos casos de CaP.

\section{TABLA \|I B. GRUPO SCREENING, SEGUNDA RONDA. DISTRIBUCIÓN SEGÚN NIVEL DE PSA BIOPSIAS REALIZADAS Y CÁNCERES DETECTADOS.}

\begin{tabular}{|l|c|c|c|c|c|c|}
\hline PSA en $\mathrm{ng} / \mathrm{ml}$ & $\begin{array}{c}\text { Número de } \\
\text { varones en } \\
\text { screening }\end{array}$ & $\begin{array}{c}\text { Número } \\
\text { referido }\end{array}$ & $\begin{array}{c}\text { Número de } \\
\text { biopsias }\end{array}$ & $\begin{array}{c}\text { Número de. } \\
\text { CaP }\end{array}$ & $\begin{array}{c}\text { P } \\
\text { de CaP } \\
(\%)\end{array}$ & $\begin{array}{c}\text { Detección } \\
\text { diferida }\end{array}$ \\
\hline$<3$ & 1527 & 254 & 155 & 14 & 0,9 & 0 \\
\hline$\geq 3<4$ & 100 & 100 & 80 & 15 & 15,0 & 2 \\
\hline$\geq 4<7$ & 89 & 89 & 68 & 8 & 9,0 & 1 \\
\hline$\geq 7<10$ & 17 & 17 & 11 & 1 & 5,9 & 0 \\
\hline$\geq 10$ & 7 & 7 & 4 & 1 & 14,3 & 0 \\
\hline Total & 1740 & 467 & 318 & 39 & 2,2 & 3 \\
\hline
\end{tabular}




\section{TABLA /I C. GRUPO SCREENING, TERCERA RONDA. DISTRIBUCIÓN SEGÚN NIVEL DE PSA, BIOPSIAS REALIZADAS Y CÁNCERES DETECTADOS.}

\begin{tabular}{|l|c|c|c|c|c|c|}
\hline PSA en $\mathrm{ng} / \mathrm{ml}$ & Número de & $\begin{array}{c}\text { Número } \\
\text { varones en } \\
\text { screening }\end{array}$ & $\begin{array}{c}\text { Número de } \\
\text { biopsias }\end{array}$ & $\begin{array}{c}\text { Número de. } \\
\text { CaP }\end{array}$ & $\begin{array}{c}P \\
\text { de CaP } \\
(\%)\end{array}$ & $\begin{array}{c}\text { Detección } \\
\text { diferida }\end{array}$ \\
\hline$<3$ & 179 & 0 & 0 & 0 & 0,0 & 0 \\
\hline$\geq 3<4$ & 8 & 8 & 5 & 0 & 0,0 & 0 \\
\hline$\geq 4<7$ & 8 & 8 & 4 & 0 & 0,0 & 0 \\
\hline$\geq 7<10$ & 1 & 1 & 1 & 0 & 0,0 & 0 \\
\hline Total & 196 & 17 & 10 & 0 & 0,0 & 0 \\
\hline
\end{tabular}

*Detección diferida = Tumores detectados en visitas sucesivas antes de la ronda siguiente (intervalo entre rondas = 4 años).

Respecto al estadío clínico de los tumores detectados (Tabla III), de los cánceres órgano confinados (T1 y T2) 102 fueron del brazo screening $(90,3 \%)$ y 24 del control $(82,8 \%), p=0,254$. Se detectaron 6 pacientes con enfermedad metastásica $(4,2 \%$ de 142$), 3$ del brazo screening $(2,7 \%)$ y 3 del control $(10,3 \%), p=0,187$.

Los resultados en cuanto a tratamiento primario se detallan en la Tabla IV. El tratamiento realizado en mayor frecuencia fue la prostatectomía radical : 47 pacientes del grupo screening $(41,6 \%)$ y 10 en el brazo control $(34,5 \%)$. Se mantuvieron en observación vigilada un total de 31 pacientes $(27,4 \%)$ en el brazo screening y 8 pacientes $(20,7 \%)$ en el brazo control.

Hasta la fecha se han producido 151 fallecimientos en los varones reclutados, 82 en el bra- zo screening y 69 en el control, de estos sólo 3 fallecimientos por $\mathrm{CaP}$, todos del brazo screening $(p=0,308)$.

\section{DISCUSIÓN}

Los programas de screening de tumores malignos se justifican cuando se centran en una enfermedad con cierta prevalencia, siempre que suponga detección en un estadio tratable, y que la actuación sobre dichos tumores disminuya la mortalidad por los mismos (12). En nuestro estudio, la mayoría de cánceres de próstata diagnosticados fueron órgano confinados. La tasa de detección fue superior durante las dos primeras rondas en los pacientes sometidos a screening $(1,7 \%$ y $2,2 \%)$. Sin embargo, el rendimiento de la biopsia prostática durante la tercera ronda fue muy inferior (concretamente nulo), datos en línea

TABLA III. DISTRIBUCIÓN DE LOS CÁNCERES DETECTADOS POR BRAZO DEL ESTUDIO Y ESTADÍO CLIINICO.

\begin{tabular}{|l|c|c|c|c|c|}
\hline & T1-T2 & T3-T4 & N1 & M1 & Total \\
\hline Screening & 102 & 5 & 3 & 3 & 113 \\
\hline Control & 24 & 2 & 0 & 3 & 29 \\
\hline Total & 126 & 7 & 3 & 6 & 142 \\
\hline
\end{tabular}


con lo publicado en estudios previos de nuestra institución (15).

Por otro lado, se observa que la tasa de detección de CaP es muy inferior en el brazo control. Aun así, la detección de tumores en el brazo control no es cero, como cabría esperar según el diseño del estudio. Esto es debido al fenómeno de contaminación (determinación de PSA y biopsia prostática fuera del protocolo del estudio, por ejemplo en la medicina de empresa o en atención primaria). Este fenómeno podría poner en peligro el poder estadístico del estudio pero, en realidad, muy pocas de estas actuaciones finalizan en biopsia prostática y en detección de cáncer $(3,16)$. A tenor de un estudio recientemente realizado en nuestro Centro (16), la contaminación de PSA en el grupo control fue calculada en 17\% (el estudio ERSPC ha sido diseñado para soportar un máximo de $20 \%$ de contaminación efectiva en el grupo control / 4 años). No obstante, existe en nuestra experiencia una diferencia en cuanto a la proporción de varones sometidos a biopsia $(21,3 \%$ en el brazo screening y $2,9 \%$ en el brazo control, $p<0.0001)$, y en las tasas de detección acumuladas $(4,7 \%$ y $1,6 \%$ respectivamente, $\mathrm{p}<0.0001$ ). Por ello, creemos poco probable que la contaminación de PSA suponga una amenaza para este estudio (16).

Además, nuestra tasa de detección es inferior a la que podemos encontrar en otros estudios. Esto puede deberse al empleo de biopsia sextante. Rabbani y cols. demostraron que el empleo de biopsia sextante puede dejar de diagnosticar hasta un $23 \%$ de los cánceres (17). Por otro lado, es posible que esta mayor detección conlleve el diagnóstico de cánceres cuya relevancia clínica pueda ser cuestionable. Master y cols. en un estudio con 317 pacientes sometidos a prostatectomía radical con un rango de 6 a 20 cilindros por biopsia, comprobaron en los pacientes con mas de 6 cilindros, el volumen tumoral era significativamente más pequeño que en los hombres con biopsia por sextantes, sugiriendo que los protocolos extendidos de toma de biopsia contribuyen a identificar más cánceres con dudosa relevancia clínica (8).

Por otro lado, la mejora en la identificación de $\mathrm{CaP}$ se asocia a una migración a estadios mas bajos y tumores con características favorables $(4,7,8)$, a diferencia de etapas anteriores a esta práctica, donde hasta un $30 \%$ de los hombres con esta enfermedad presentaban metástasis en el momento diagnóstico (3). Aus y cols. encontraron un aumento en el riesgo de ser diagnosticado de $\mathrm{CaP}$ en un 1,8 a 2 veces con el screening, además una mayor probabilidad de enfermedad metastásica en varones no sometidos a screening. No obstante los autores no encontraron diferencias en cuanto a supervivencia (18). En el presente estudio no hubo diferencias en cuanto al hallazgo de enfermedad metastásica entre ambos brazos de estudio.

En nuestro Centro se ha preferido la cirugía radical de próstata como tratamiento primario de cánceres órgano confinados. Llama la atención la elevada tasa de pacientes sometidos a observación vigilada (la segunda en importancia, con $27,5 \%$ de todos los tumores detectados). Esto podría interpretar-

TABLA IV. DISTRIBUCIÓN DE LOS CÁNCERES DETECTADOS SEGÚN TRATAMIENTO INICIAL.

\begin{tabular}{|l|c|c|c|c|c|c|c|}
\hline \multicolumn{3}{|c}{ Screening } & \multicolumn{5}{c}{ Control } \\
\hline & T1-T2 & T3-T4 & M1 & T1-T2 & T3-T4 & M1 & total \\
\hline Solo cirugía & 47 & 0 & 0 & 10 & 0 & 0 & 57 \\
\hline Solo radioterapia & 14 & 3 & 0 & 4 & 0 & 0 & 21 \\
\hline Solo hormonoterapia & 0 & 1 & 3 & 1 & 0 & 3 & 8 \\
\hline Radioterapia + hormonoterapia & 6 & 3 & 0 & 0 & 0 & 0 & 9 \\
\hline Observación vigilada & 31 & 0 & 0 & 6 & 2 & 0 & 39 \\
\hline No conocido & 5 & 0 & 0 & 3 & 0 & 0 & 8 \\
\hline
\end{tabular}


se como cierta limitación del estudio, ya que la filosofía del screening del CaP no se limita a la detección, sino al tratamiento (siempre que sea posible) de los tumores encontrados.

A pesar de que el tiempo de seguimiento de los varones estudiados es alto (media de 8,9 años), tan sólo un 3,5\% de ellos han fallecido (por múltiples causas). Por ello, el presente estudio no permite aportar todavía datos que permitan demostrar un cambio en la mortalidad cáncer específica tras el screening. Este dato hace pensar que se necesitan tamaños muestrales y tiempos de seguimiento muy importantes para que estos estudios den sus frutos. Confiamos que en un futuro no muy lejano esta incógnita quede resuelta.

\section{CONCLUSIONES}

Se observa una tendencia no significativa hacia estadíos clínicos mas precoces en los pacientes sometidos a screening. El tratamiento de elección en los pacientes con CaP fue la cirugía radical. Aún con el seguimiento actual de esta serie (cercano a los 9 años), todavía no se han observado en nuestro Centro diferencias en cuanto a la mortalidad por $\mathrm{CaP}$ entre los dos brazos del estudio.

\section{BIBLIOGRAFÍA y LECTURAS RECOMENDADAS (*lectura de interés $y^{* *}$ lectura fundamental)}

*1. MARTIN, R.; GUNNELL, D.; HAMDY, F. y cols.: "Continuing controversy over monitoring men with localized prostate cancer: A systematic review of programs in the prostate specific antigen era". J. Urol., 176: 439, 2006.

2. JEMAL, A.; SIEGEL, R.; WARD, E. y cols.: "Cancer statistics 2006". CA Cancer J. Clin., 56: 106, 2006.

3. LUJAN, M.; PASCUAL, C.; ANGULO, J. y cols.: "Cribado del cáncer de próstata. La controversia". Rev. Urol., 7: 3, 2006.

*4. LUJÁN, M.; PÁEZ, A.; LLANES, L. y cols.: "Rasgos diferenciales del cáncer de próstata procedentes de un programa de detección precoz frente al detectado en una consulta de urología". Actas Urol. Esp., 24: 640, 2000.

5. SWANSON, G.: "Management of locally advanced prostate cancer: Past, present, future". J. Urol., 176: 34, 2006.

*6. MAKAROV, D.; CARTER, H.B.: "The discovery of prostate specific antigen as a biomarker for the early detection of adenocarcinoma of the prostate". J. Urol., 176: 2383, 2006.
7. MENG, M.; ELKIN, E.; DUCHANE, J. y cols.: "Capsure investigators: Impact of increased number of biopsies on the nature of prostate cancer identified". J. Urol., 176: 63, 2006.

8. MASTER, V.; CHI, T.; SIMKO, J. y cols.: "The independent impact of extended pattern biopsy on prostate cancer stage migration". J. Urol., 174: 1789, 2005.

9. LUJÁN, M.; PÁEZ, A.; SANCHEZ, E. y cols.: "Prostate specific antigen variation in patients without clinically evident prostate cancer". J. Urol., 162: 1311, 1999.

*10. SMITH, R.A.; COKKINIDES, V.; EYRE, H.J.: "American cancer society guidelines for the early detection of cancer 2006." CA Cancer J. Clin., 56: 11, 2006.

11. STAMEY, T.A.; CALDWELL, M.; McNEAL, J.E. y cols.: "The prostate specific antigen era in the Unite States is over for prostate cancer: What happened in the last 20 years?". J. Urol. 172: 1297, 2004.

**12. DE KONING, H.J.; AUVINEN, A.; BERENGUER, A. y cols.: "For the ERSPC and the PLCO Trials (the International Prostate Cancer Screening Trials Evaluation Group)Large-scale randomized prostate cancer screening trials: program performances in the European randomized screening for prostate cancer trial and the prostate, lung, colorectal and ovary cancer trial". Int. J. Cancer. 97: 237, 2002.

13. http://www.epi.bris.ac.uk/protect/, último acceso el 14/1/2007.

14. BERENGUER, A.; LUJAN, M.; PÁEZ, A. y cols.: "The spanish contribution to the european randomized study of screening for prostate cancer". BJU Int., 92: 33, 2003.

15. LUJAN, M.; PÁEZ, A.; SANTOJA, C. y cols.: "Prostate cancer detection and tumor caracteristics in men with múltiple biopsy sessions". Prostate Cancer Prostatic Dis., 7: 238, 2004.

**16. LUJAN, M.; PÁEZ, A.; PASCUAL, T. y cols.: "Extent of prostate specific antigen contamination in the spanish section of the european randomized study of screening for prostate cancer (ERSPC)". Eur. Urol., 50: 1234, 2006.

17. RABBANI F, STROUMBAKIS N, KAVA BR, Cookson MS, Fair WR. Incidence and clinical significance of false-negative sextant prostate biopsies. J. Urol. 159:1247, 1998.

*18. AUS, G.; BERGDAHL, S.; LODDING, P. y cols.: "Prostate cancer screening decreases the absolute risk of being diagnosed with advanced prostate cancer-results from a prospective, population-based randomized controlled trial". Eur. Urol., 51: 659, 2007. 